\title{
ALINEACIÓN DE LOS SISTEMAS DE INFORMACIÓN CON LA ESTRATEGIA DE GOBIERNO EN LÍNEA
}

\author{
Paula Andrea Pérez Gómez \\ Universidad Católica de Colombia
}

\section{Resumen}

Este artículo busca plantear la interpretación jurídica de la imposición establecida por el Legislador en el Artículo 17 literal d de la Ley 1712 de 2014, que trata de la alineación que debe existir entre la estrategia de Gobierno en línea y los sistemas informáticos implementados por los sujetos obligados por el Artículo 5 de la misma Ley. Este requisito fue instituido con el fin de garantizar que tales sistemas sean una herramienta para promover el acceso a la información pública. Con el propósito de identificar los requerimientos que los sujetos obligados deben cumplir para lograr dicha "alineación”, se estudiará el derecho de acceso a la información pública, la estrategia de Gobierno en línea y la relación de esta con los deberes de los sujetos obligados.

Palabras clave: acceso, información, sistemas informáticos, Gobierno en línea, TIC (tecnologías de la información y la comunicación), publicidad.

La autora: estudiante de décimo semestre de Derecho de la Universidad Católica de Colombia, auxiliar de investigación y miembro del grupo de investigación en Derecho Público y TIC. Correo electrónico: paula.pperez.gomez@gmail.com

Recibido: 17 de agosto de 2016; evaluado: 14 de noviembre de 2016; aceptado: 25 de noviembre de 2016. 


\title{
ALIGNMENT OF INFORMATION SYSTEMS WITH ONLINE GOVERNMENT STRATEGY
}

\author{
Paula Andrea Pérez Gómez \\ Universidad Católica De Colombia
}

\begin{abstract}
This article aims to present the legal interpretation of the requirement established by the Legislator in the Article 17, paragraph d, of Law 1712 of 2014, which deals with the alignment that must exist between the online government strategy and the computer systems implemented by the subjects obligated by Article 5 of the same Law. This requirement was established in order to ensure that such systems constitute a tool to promote access to public information. In order to identify the requirements that the obligated subjects must meet in order to achieve this "alignment," this paper examines the right of access to public information as well as the online government strategy and its relationship with the duties of the obligated subjects.
\end{abstract}

Keywords: access, information, computer systems, online government, ICTs (information and communication technologies), advertising.

About the author: Tenth semester law student at the Universidad Católica de Colombia, research assistant, and member of the Research Group on Public Law and ICT. Email: paula.pperez.gomez@ gmail.com

Received: August 17, 2016; evaluated: November 14, 2016; accepted: November 25, 2016. 


\section{Introducción}

El uso de las tecnologías en pro del acceso a la información, la creación de la política pública del Gobierno abierto, la implementación de la Ley de transparencia y acceso a la información pública y la aplicación de la estrategia de Gobierno en línea son el resultado de un esfuerzo interdisciplinario en busca de la garantía del derecho de acceso a la información pública, la transparencia y el perfeccionamiento de la relación Administración-administrado.

Existen estudios doctrinales, tanto nacionales como internacionales, que analizan cuestiones relacionadas con la implementación de las TIC para mejorar la prestación de servicios públicos y el impacto del uso de las tecnologías en la realidad social, entre otros temas que exigen hermenéutica jurídica dada su importancia para la sociedad.

Al respecto, se pueden señalar doctrinantes como Lorenzo Cotino Hueso, profesor titular de Derecho Constitucional de la Universitat de València y coordinador de la Red Derecho TIC, quien no solo ha publicado libros sobre el derecho de acceso a la información pública y TIC, sino que también ha hecho ponencias; Antônio Carlos Efin y Cinthia Obladen de Almendra Freitas, autores del libro Direito e questões tecnológicas aplicados no desnvolvimento Socia volúmenes 1 y 2; Jairo Becerra, director de investigación de la Universidad Católica de Colombia, coautor del libro La responsabilidad del Estado por la utilización de las tecnologías de la información y la comunicación, entre otros.

Al observar el literal d, Artículo 17 de la Ley 1712 de 2014, Ley de transparencia y acceso a la información pública, surgió la necesidad de interpretar la exigencia hecha por el Legislador en relación con la alineación que debe existir entre la estrategia de Gobierno en línea y los sistemas informáticos implementados por los sujetos obligados por la Ley. Esto se resume en la pregunta: ¿cuáles son las exigencias o los elementos que debe cumplir el sujeto obligado para que su sistema informático se encuentre alineado con la estrategia de Gobierno en línea?

Para dar respuesta a esta pregunta, se utilizará el método deductivo-argumentativo y el artículo se dividirá en cuatro capítulos: el primero, destinado al análisis sobre la importancia del derecho de acceso a la información pública y su protección por medio de la Ley 1712 de 2014; el segundo, dirigido al estudio de los deberes de la Administración pública respecto a la publicidad de la información —entre las que 
se encuentra la exigencia objeto de análisis en este artículo—y a la exposición de la estrategia de Gobierno en línea y del marco normativo que la comprende; el tercero está relacionado con los sistemas de información y el uso de las TIC y el cuarto, "Alineación del sistema de información con la estrategia de Gobierno en línea".

\section{Derecho al acceso a la información pública en Colombia}

El derecho al acceso a la información pública tiene como objetivo principal "el derecho de las personas de mejorar su calidad de vida". ${ }^{1}$ Esta frase hace referencia a la relación que tiene el derecho de acceso a la información con otros derechos fundamentales, que surge como consecuencia del poder de la información como herramienta que permite a las personas hacer efectivos sus derechos.

La alineación que exige el Artículo 17 de la Ley 1712 de 2014 con respecto a los sistemas informáticos (SI) y el Gobierno en línea tiene como finalidad hacer que los Sí utilizados por los sujetos obligados sean herramientas para garantizar el acceso a la información pública, por lo que es pertinente analizar la protección de este derecho en el ordenamiento jurídico colombiano y su importancia.

\subsection{Protección del derecho al acceso a la información y su importancia}

El reconocimiento de la importancia del derecho al acceso a la información pública por parte de la Administración se ha reflejado en la expedición de leyes y la creación de políticas públicas en busca de la garantía de este derecho. A continuación se expondrá el marco constitucional del derecho de acceso a la información pública y su desarrollo legal, con el fin de observar la protección jurídica de este derecho en Colombia.

En la Constitución Política de 1991 se evidencia la protección del derecho al acceso a la información pública en sus Artículos 20,74 y 270, en los cuales se hacen afirmaciones muy importantes. El Artículo 20 de la Constitución menciona: "Se garantiza a toda persona la libertad de informar y recibir información veraz e imparcial"; el Artículo 74 establece: "Todas las personas tienen derecho a acceder a los documentos públicos salvo los casos que establezca la ley", lo cual significa que

1 Ernesto Villanueva, Derecho de acceso a la información pública en Latinoamérica (Ciudad de México: Instituto de Investigaciones Jurídicas, UNAM, 2004), 14. 
la excepción a la regla general de que las personas tienen acceso a la información pública es cuando se está ante la información de carácter reservado; por último, el Artículo 270 predica: "La ley organizará las formas y los sistemas de participación ciudadana que permitan vigilar la gestión pública que se cumple en los diversos niveles administrativos y sus resultados".

De las frases transcritas se puede señalar que el derecho al acceso a la información pública no es un derecho absoluto, ya que la ley puede consagrar los casos en los que no es aplicable. También se concluye que, además de ser un derecho fundamental que se debe garantizar a todas las personas, es un medio control de la gestión pública administrativa estrechamente relacionado con el derecho fundamental al derecho de petición, consagrado en el Artículo 23 de la Constitución Política.

Las normas que protegen el acceso a la información pública "permiten a los individuos y grupos que tengan acceso a las políticas mediante las cuales el gobierno toma decisiones respeto a proyectos de salud, educación y vivienda e infraestructura y las razones que sustentan tales decisiones". ${ }^{2}$ Por eso, al tener los conocimientos por medio de la publicación de la información, los ciudadanos adquieren un papel en la sociedad que les posibilita ejercer sus derechos.

En Colombia, por ejemplo, la Ley 1712 de 2014 fue creada como "una norma de carácter estatutario que unifica criterios de transparencia y acceso a la información bajo un mismo marco". Este mandato trae consigo innovaciones importantes para el ordenamiento jurídico colombiano, entre ellas, haber consagrado como derecho fundamental autónomo el derecho de acceso a la información pública y darle el reconocimiento que se merece como consecuencia de su "carácter instrumental", pues la garantía de este derecho implica "la verdadera eficacia de los derechos que debe garantizar la administración". ${ }^{3}$

Cabe mencionar que la protección legal del derecho al acceso a la información no inició con la Ley 1712 de 2014. Han existido leyes anteriores en las que, aunque no hubo un desarrollo específico de este derecho, sí existió mención acerca de su garantía. La Ley 57 de 1985 ordenó la publicidad de los actos y documentos oficiales; dedica todo su Título II al acceso de los ciudadanos a los documentos:

The Carter Center, Access to Information: A Key to Democracy (Atlanta: Autor, 2002), 5.

Eduardo Gamero Casado y Juliana Valero Torrijos, coords., La Ley de administración electrónica (Pamplona: Aranzadi, 2008), 59. 
Toda persona tiene derecho a consultar los documentos que reposen en las oficinas públicas y a que se le expida copia de los mismos, siempre que dichos documentos no tengan carácter reservado conforme a la Constitución o la ley, o no hagan relación a la defensa o seguridad nacional. ${ }^{4}$

\subsection{Importancia del derecho al acceso a la información pública}

El derecho de acceso a la información es un instrumento que permite hacer efectiva la transparencia. ${ }^{5}$ Un Estado social de Derecho como el Estado colombiano, según el Artículo 1 de nuestra Constitución Política, requiere tener una Administración transparente para poder cumplir sus fines, entre los cuales se destacan:

[...] servir a la comunidad, promover la prosperidad general y garantizar la efectividad de los principios, derechos y deberes consagrados en la Constitución; facilitar la participación de todos en las decisiones que los afectan y en la vida económica, política, administrativa y cultural de la Nación; defender la independencia nacional, mantener la integridad territorial y asegurar la convivencia pacífica y la vigencia de un orden justo. ${ }^{6}$

El acceso a la información posibilita que las personas estén enteradas de las actuaciones de los administradores por medio de la publicidad de la información, por lo que el Gobierno rinde cuentas de sus actuaciones. Una muestra de la intención del Estado por garantizar este derecho en pro de la transparencia es la Ley de acceso a la información pública de 2014 que, por medio de la garantía del principio de publicidad que funciona en esta ley como un instrumento para promover el acceso a la información, aseguró el derecho del acceso a la información hacia la anhelada transparencia que se busca desde la Constitución de 1991.

El derecho de acceso a la información también constituye una herramienta esencial para combatir la corrupción y mejorar la calidad de nuestra democracia, ${ }^{7}$ ya

4 Congreso de la República Colombia, Ley 57 de 1985, "Por la cual se ordena la publicidad de los actos y documentos oficiales" (Bogotá: Diario Oficial, 5 de julio de 1985), art. 12.

5 María Concepción Torres Díaz, "La fundamentalidad del derecho de acceso a la información pública: análisis crítico desde el marco constitucional de la participación ciudadana" en El paradigma del Gobierno abierto. Retos y oportunidades de la participación, transparencia y colaboración, eds. Lorenzo Cotino, José Luis Sahuquillo y Loreto Corredoira (Madrid: Universidad Complutense de Madrid y ObservatorioTICs-Cyberlaw Clinic, 2015), 117.

6 República de Colombia, Constitución Política (Bogotá: Legis, 1991), art. 2.

7 Organización de Estados Americanos [OEA], "Relatoría especial para la libertad de expresión 2007". http://cidh.oas.org/relatoria/section/Estudio\%20Especial\%20sobre\%20el\%20derecho\%20de\%20 Acceso\%20a\%20la\%20Informacion. pdf (acceso julio 20 de 2016). 
que una de las consecuencias de garantizarlo es que las autoridades tengan que rendir cuentas sobre sus actuaciones. Contar con procedimientos que garanticen el acceso a la información en poder del Estado contribuye al control de la gestión estatal, ${ }^{8}$ pues permite que la comunidad tenga conocimiento tanto de las políticas públicas promovidas por la Administración, como de aspectos en los que no se ha manifestado, para requerirla por las vías establecidas en la ley y lograr que se garanticen los derechos que han dejado de protegerse. Además, facilita el control sobre los máximos detentadores de información y también garantiza derechos en la dimensión privada, en la medida en que señala el camino para satisfacer determinadas pretensiones. ${ }^{9}$ Por medio de este derecho se garantiza no solo el derecho a la información, sino a todos los que surgen de él. Respecto a ello, la Corte Constitucional, en Sentencia C-274 de 2013, mencionó que por medio del acceso a la información pública se hace efectivo el ejercicio de otros derechos constitucionales, ya que permite conocer las condiciones necesarias para su realización.

En un estudio especial sobre el derecho al acceso a la información pública, desarrollado por la OEA, se afirmó que "la democracia consiste en la habilidad de los individuos de participar efectivamente en la toma de decisiones que los afecten; y esta participación depende de la información con la que se cuente", ${ }_{10}^{10}$ por lo que el derecho al acceso a la información tiene la función de garantizar la participación ciudadana.

Algunos doctrinantes han manifestado su preocupación sobre lo que denominan "crisis de legitimidad de los procedimientos de la democracia" y destacan que en la actualidad "se demanda más democracia y más participación, y que esto solo se puede lograr permitiendo que los ciudadanos participen en los procesos de toma de decisiones", 11 como ya hemos dicho, esto solo se logra si los ciudadanos tienen acceso a la información y a los sistemas informáticos que se utilicen con miras a impulsar la participación ciudadana.

En conclusión, la importancia del derecho de acceso a la información pública se puede sintetizar en su papel para la consagración de otros derechos, su uso para

8 Federación Iberoamericana de Ombudsman, XIII Informe sobre derechos humanos. transparencia e información pública (Madrid: Trama editorial, 2015), 61.

9 Torres Díaz, "La fundamentalidad del derecho de acceso a la información pública", 117.

10 Organización de Estados Americanos [OEA], "Relatoría especial para la libertad de expresión 2007", 45.

11 Jacinto Porro Gutiérrez, Instrumentos y procesos de participación ciudadana en España y Marruecos (Madrid: Dykinson, 2012), 15. 
prevenir la corrupción, su importancia como medio de control de la actuación de la Administración y su función como medio para garantizar la participación democrática y la transparencia.

\subsection{Uso de las TIC para la protección del derecho al acceso a la información pública}

En los últimos tiempos, ha sido evidente el papel de las tecnologías de la información como medio para garantizar el acceso a la información pública. La contribución de las TIC no solo se ha visto reflejada en la creación de un Gobierno más transparente y participativo, sino que su implementación "puede significar mejoras en los procesos internos que trascienden hacia un Estado mucho más organizado", ${ }^{12}$ debido a que al emplear estas herramientas se facilita la condensación y el flujo de la información.

Para reconocer la importancia del uso de las TIC, primero es necesario definirlas. Utilizaremos el concepto que surgió de una investigación publicada en la revista de comunicaciones de origen vasco ZER, en la que se analizó y recopiló la definición de TIC y se concluyó que son:

Dispositivos tecnológicos que permiten editar, producir, almacenar, intercambiar y transmitir datos entre diferentes sistemas de información que cuentan con protocolos comunes. Estas aplicaciones, que integran medios de informática, telecomunicaciones y redes, posibilitan tanto la comunicación y colaboración interpersonal (persona a persona) como la multidireccional (uno a muchos o muchos a muchos). Dichas herramientas desempeñan un papel sustantivo en la generación, intercambio, difusión, gestión y acceso al conocimiento. ${ }^{13}$

Con un concepto general de las TIC podemos darnos una idea de la importancia de su uso en la relación Administración-administrado y de cómo esto es consecuencia del papel de las tecnologías en un mundo globalizado que requiere de medios de comunicación de carácter electrónico y masivo.

12 Jesús Alberto Andrade Castro y Yaskelly Yedra Hernández, Sistemas transparentes para gobiernos electrónicos eficientes (Maracaibo: Red Enlace, 2007), 82.

13 Juan Cristóbal Cobo Romaní, "El concepto de tecnologías de la información. Benchmarking sobre las definiciones de las TIC en la sociedad del conocimiento", Zer: Revista de estudios de comunicación 14, núm. 27 (2009): 312. 
Respecto al tema de la regulación de las TIC y su relación con el Derecho se ha dicho:

En cada uno de los nuevos escenarios del sector de las TIC, el Derecho ha entrado, o está en proceso de hacerlo, a comprender y regular de una forma un tanto abrupta e improvisada la interacción que se presenta entre el Estado, los ciudadanos y los proveedores de servicios de telecomunicaciones. ${ }^{14}$

Todo esto en razón a las numerosas regulaciones nacionales e internacionales que han tratado el tema, entre las que se encuentran la Ley 54 de 1973, "Por medio de la cual se aprueba el Acuerdo relativo a la Organización Internacional de Telecomunicaciones por Satélite 'Intelsat', celebrado en Washington, el día 20 de agosto de 1971"; la Ley 46 de 1985, "Por medio de la cual se aprueban el 'Convenio de Telecomunicaciones'; la Ley 1341 de 2009, también llamada Ley de las TIC y las leyes que no regulan directamente el tema, pero sí usan las TIC como medio para el logro de sus propósitos (el debido proceso y el acceso a la información pública). Ejemplo de estas leyes son el Código General del Proceso (cuyo Artículo 103 destaca que en todas las actuaciones judiciales deberá procurarse el uso de las tecnologías de la información y las comunicaciones en la gestión y el trámite de los procesos judiciales, con el fin de facilitar y agilizar el acceso a la justicia, así como ampliar su cobertura), el Código de Procedimiento Administrativo y de lo Contencioso Administrativo (cuyo Artículo 3 predica que para el cumplimiento de los principios de publicidad y celeridad se incentivará el uso de las tecnologías) y la Ley de transparencia y del derecho de acceso a la información pública que promueve el uso de las TIC para la garantía del derecho de acceso a la información.

Según la Ley 1712 de 2014, todos tienen derecho al acceso a la información pública; sin embargo, hacer efectivo este derecho para toda la población colombiana es imposible sin el uso de las tecnologías de la información y la comunicación, ya que aun cuando existen algunas personas que no tienen la capacidad o los elementos para acceder por medios tecnológicos, es un hecho que con los medios electrónicos se puede dar a conocer la información a un mayor número de personas, como lo han mostrado los boletines trimestrales del MinTIC. ${ }^{15}$

Jairo Becerra, et. al. , El Derecho y las tecnologías de la información y la comunicación (TIC) (Bogotá: Universidad Católica de Colombia, 2015), 9.

15 MinTIC, "Boletín trimestral de las TIC. Cifras primer trimestre 2016)", http://colombiatic.mintic.gov.co/602/ articles-15639_archivo_pdf. pdf (acceso agosto 3 de 2016), 7, 13, 18, 41. 
Respecto al pronunciamiento internacional sobre el uso de las tecnologías con el fin de promover la transparencia y el acceso a la información pública, el 8 de diciembre de 2009, la Casa Blanca publicó la directiva de Open Government, ${ }^{16}$ que requiere a las agencias federales para que den pasos inmediatos para alcanzar la transparencia, la participación y la colaboración.

En la Directiva se habla de lograr un Gobierno abierto por medio de la publicación de la información en línea y el uso de la tecnología moderna. ${ }^{17}$ Sobre esta política pública se han pronunciado algunos doctrinantes como el doctor Lorenzo Cotino Hueso, quien señaló que al aplicar la política pública de Gobierno abierto se logra un sistema democrático participativo; por ello, afirma que el Gobierno abierto es transparencia que procura calidad en los servicios públicos, la configuración de servicios públicos electrónicos para el usuario y el uso de las tecnologías como las redes sociales para difundir información e incentivar el ahorro en campañas institucionales. ${ }^{18}$ Así se logra un mayor contacto con los ciudadanos a menor costo y se garantiza no solo el acceso a la información, sino la participación de la sociedad, lo que permite controlar socialmente la gestión de la Administración.

\section{Deberes de la Administración pública respecto a la publicidad de la información}

Analizar el deber de publicar la información por parte de la Administración es necesario porque "en principio, la dimensión constitucional de la información pública deriva de múltiples disposiciones entre las que se encuentra la publicidad de los actos y las normas". ${ }^{19}$ Esto explica el papel de la publicidad en función de la promoción del acceso a la información, pues es el acto por el que se dan a conocer las actuaciones y la información de la Administración.

16 The White House, "Open Government Initiative", https://www.whitehouse.gov/open (acceso agosto 17 de 2016).

17 Peter R. Orszag, "Memorandum for the Heads of Executive Departments and Agencies, de 8 de diciembre de 2009", https://www.whitehouse.gov/open/documents/open-government-directive (acceso agosto 17, 2016).

18 Lorenzo Cotino Hueso, "Derechos fundamentales de acceso a la información pública y participación ciudadana, regulación constitucional, estatutaria y legal" (ponencia presentada en el seminario El Gobierno abierto en el Estatuto de Autonomía y su desarrollo. Especial atención a la futura ley de transparencia, buen gobierno y participación ciudadana de la Comunitat Valenciana, Valencia, 30 de septiembre, 2014).

19 Catalina Ruiz-Rico, Tendencias legales hacia la calidad democrática del sistema constitucional (Madrid: Dykinson, 2014), 13. 
La presencia de la publicidad es vital para la consagración del Estado social de Derecho, tanto, que Kant y Weber resaltaron su importancia: "Kant señalando que son injustas todas las acciones que se refieren al derecho de otros hombres cuyos principios no soportan ser publicados y Weber diciendo que el gobierno burocrático es, por su misma tendencia, un gobierno que excluye la publicidad". ${ }^{20}$

Ahora bien, puesto que el propósito de este trabajo es comprender la exigencia de los sujetos obligados a alinear los sistemas informáticos (SI) y la estrategia de Gobierno en línea, es vital exponer las obligaciones que surgen del principio de la divulgación proactiva de la información consagrado en la Ley 1712 de 2014 y hacer énfasis en las obligaciones en relación con los sistemas de información como herramientas para el acceso a la información pública.

\subsection{Publicidad de la información en la Ley 1712 de 2014}

La Sentencia C-274 de 2014 —por medio de la cual se hace la revisión constitucional del Proyecto de Ley Estatutaria 228 de 2012 Cámara, 156 de 2011 Senado, por la que se crea Ley de transparencia y del derecho de acceso a la información pública nacional- menciona que su objeto e importancia tienen sustento en los Artículos 15, 20, 23, 74 y 78 de la Constitución Política.

Entre las finalidades y los principios de la Ley se encuentra el de transparencia, sobre el cual se ha dicho que "es el sustento del Estado social de Derecho y la democracia que está ligado con la publicidad de las normas y los actos"; 21 este es el que permite el desarrollo de los Estados y genera confianza de las personas hacia las actuaciones de la Administración.

Asimismo, la Ley 1712 plantea que, en virtud del principio de transparencia, toda la información de los sujetos obligados se presume pública y por esta razón se deberá garantizar el acceso a la información, principio que es desarrollado dentro del articulado de la misma Ley. En ella se imponen deberes en relación con la publicidad de la información bajo los siguientes criterios:

20 Lorenzo Cotino Hueso, "Teoría y realidad de la transparencia pública en Europa", http://docplayer. es/11550709-Teoria-y-realidad-de-la-transparencia-publica-en-europa-lorenzo-cotino-hueso-2003. html (acceso agosto 25, 2016), 7.

21 Salvador Nava, Issa Luna y Ernesto Villanueva, Derecho de acceso a la información pública parlamentaria (Ciudad de México: Porrúa, 2006), 23. 
- Disponibilidad de la información. De acuerdo con el Artículo 7, la disponibilidad de la información se resume en dos obligaciones: i) deberá estar a disposición del público la información a la que hace referencia la Ley de transparencia y acceso a la información pública por medios físicos, remotos o locales de comunicación electrónica; esto implica la disposición de la información en la página web para que los administrados accedan a la información de manera directa o mediante impresiones, y ii) se deberá proporcionar apoyo a los usuarios que lo requieran y proveer todo tipo de asistencia respecto a los trámites y servicios que presten.

- Criterio diferencial de accesibilidad. Según el Artículo 8, con el propósito de garantizar el acceso a la información pública a poblaciones específicas, las autoridades deberán: i) divulgar la información pública en diversos idiomas y lenguas y elaborar formatos alternativos comprensibles para poblaciones específicas, y ii) asegurar el acceso a esa información a los grupos étnicos y culturales del país y adecuar los medios de comunicación para que faciliten el acceso a las personas que se encuentran en situación de discapacidad.

- Información mínima del sujeto obligado. La Ley de transparencia y acceso a la información pública exige a los sujetos obligados, inscritos en su Artículo 5, a publicar información mínima determinada por la Ley de carácter imperativo y de manera proactiva en los sistemas de información del Estado o herramientas que lo sustituyan. Dicha información está inscrita taxativamente en los Artículos 9 y 11 de la Ley, que respectivamente tratan de la estructura del sujeto obligado (información que según el parágrafo del Artículo 10 deberá actualizarse al menos cada mes) y la información mínima respecto a servicios, procedimientos y funcionamiento del sujeto obligado.

- Publicidad de la contratación. El Artículo 10 impone el deber de publicar y actualizar la información referente a las contrataciones sometidas al régimen de contratación estatal, mínimo cada mes, por medio electrónico institucional. Este deber permite el acceso directo a la información correspondiente al proceso contractual en aquellos que se encuentren sometidos a dicho sistema, sin excepción.

- Adopción de esquemas de publicación. Según el Artículo 12, todo sujeto obligado deberá adoptar y difundir de manera amplia su esquema de publicación, dentro de los seis meses siguientes a su entrada en vigencia. El esquema 
será difundido mediante su sitio web y, en su defecto, en los dispositivos de divulgación existentes en su dependencia, incluidos boletines, gacetas y carteleras. La Ley también indica en el mismo Artículo lo que deberá establecer el esquema de publicación.

- Registros de activos de información. En virtud del Artículo 13, todo sujeto obligado deberá crear y mantener actualizado el registro de activos de información y hacer un listado de todas las categorías de información publicada por el sujeto obligado, todo registro publicado y todo registro disponible para ser solicitado por el público.

- Información publicada con anterioridad. El Artículo 14, corregido por el Artículo 1 del Decreto 1862 de 2015, exige a los sujetos obligados garantizar y facilitar a los solicitantes, de la manera más sencilla posible, el acceso a toda la información previamente divulgada. Esto implica que las respuestas a las solicitudes hechas con anterioridad deberán hacerse públicas de modo proactivo en el sitio web del sujeto obligado o en los dispositivos de divulgación existentes de la dependencia.

- $\quad$ Programa de gestión documental. El Artículo 15 impone el deber a los sujetos obligados a adoptar un programa de gestión documental en el cual se establezcan los procedimientos y lineamientos necesarios para la producción, distribución, organización, consulta y conservación de los documentos públicos, dentro de los seis meses siguientes a la entrada en vigencia de la presente Ley.

- Archivos. El Artículo 16 dice que los sujetos obligados deben asegurarse de que existan dentro de sus entidades procedimientos claros para la creación, gestión, organización y conservación de sus archivos.

\subsection{Obligación de los sujetos obligados por la Ley 1712 de 2014 en relación con los sistemas de información}

El principio de transparencia inscrito en la Ley 1712 plantea que se deberá proporcionar y facilitar el acceso a la información por los medios que establece la Ley. Unos de estos medios que pueden ser implementados por los sujetos obligados son los sistemas de información sobre los cuales el Artículo 17 hace las siguientes exigencias con el propósito de garantizar su efectividad para promover el acceso a la información pública: 
- Que se encuentren alineados con los procedimientos y articulados con los lineamientos establecidos en el programa de gestión documental de la entidad.

- Que gestionen la misma información que se encuentre en los sistemas administrativos del sujeto obligado.

- Que, en el caso de la información de interés público, exista una ventanilla en la que se pueda acceder a la información en formatos y lenguajes comprensibles para los ciudadanos.

- Que estén alineados con la estrategia de Gobierno en línea o de la que haga sus veces. Respecto a esta última exigencia, el párrafo 3 del Artículo 9 dice que, en cuanto a la publicación y divulgación de la información, los sujetos obligados deberán observar lo establecido por la estrategia de Gobierno en línea o la que haga sus veces.

\section{La estrategia de Gobierno en línea y los sistemas informáticos}

Para dar respuesta a la pregunta sobre cuáles son las exigencias o los elementos que debe cumplir el sujeto obligado para que su sistema informático se encuentre alineado con la estrategia de Gobierno en línea, primero se deben entender los conceptos de Gobierno en línea y de sistema informático. Por eso, este capítulo tiene dos objetivos: el primero, explicar qué es la estrategia de Gobierno en línea y exponer sus avances y el segundo, presentar los conceptos de sistema de información y sistema informático, con el fin de evidenciar que como se exige la alineación de los sistemas de información con la estrategia de Gobierno en línea y que la estrategia implementa las tecnologías de la información y la comunicación, la Ley ya no estaría hablando de sistemas de información, sino de sistemas informáticos.

\subsection{Estrategia de Gobierno en línea}

Ya que Gobierno en línea es una estrategia de gobierno electrónico es oportuno precisar el concepto de este último. El libro Gobierno electrónico: un signo de inclusión digital y poder popular lo define como "la simple adopción de las infotecnologías 
por parte de la gestión pública gubernamental como una de las vías a través de la que se conectan e interactúan el gobierno con otras organizaciones y personas". ${ }^{22}$

Por su parte, el sitio oficial de la estrategia de Gobierno en línea define a la misma como la estrategia de gobierno electrónico en Colombia, que busca construir un Estado más eficiente, más transparente y más participativo gracias al uso de las tecnologías de la información y la comunicación. ${ }^{23}$

Los propósitos de la estrategia son: prestar servicios de calidad en línea, darle un uso estratégico a la tecnología para hacer más eficaz la gestión administrativa, proteger los datos de los ciudadanos, atender la privacidad y garantizar la seguridad de la información, ${ }^{24}$ así como impulsar y facilitar acciones requeridas para avanzar en los objetivos de desarrollo sostenible. ${ }^{25}$

\subsection{Marco normativo de la estrategia de Gobierno en línea}

\subsubsection{Constitución Política de Colombia}

A pesar de que en 1991 aún no se hablaba de la estrategia de Gobierno en línea, sí se apreciaban fundamentos de la misma en la Constitución, en los Artículos 20 y 206.

El Artículo 20 es el fundamento del derecho a la información y dice: "Se garantiza a toda persona la libertad de expresar y difundir su pensamiento y opiniones, la de informar y recibir información veraz e imparcial, y la de fundar medios masivos de comunicación". A su vez, es base para el derecho al acceso a la información, que es garantizado mediante las TIC con la implementación de la estrategia de Gobierno en línea.

El Artículo 206 predica: "La función administrativa está al servicio de los intereses generales y se desarrolla con fundamento en los principios de igualdad, moralidad, eficacia, economía, celeridad, imparcialidad y publicidad, mediante la descentralización, la delegación y la desconcentración de funciones".

22 José Ramírez, Deslisireth Rincón y María Romero, "Gobierno electrónico: un signo de inclusión digital y poder popular, Revista de Ciencias Sociales 16, núm 4 (2010): 715.

23 República de Colombia, "Estrategia de Gobierno en línea", http://estrategia.gobiernoenlinea.gov.co/623/ w3-propertyvalue-7650. html (acceso agosto 26, 2016).

24 República de Colombia, "Estrategia de Gobierno en línea".

25 República de Colombia, "Declaración de compromiso con la agenda post 2015 —ODS", http://estrategia. gobiernoenlinea.gov.co/623/articles-7929_agenda_ods. pdf (acceso julio 26, 2016). 


\section{2. 2. Documento Conpes 2790 de 1995}

Este instrumento presenta:

[... la la estrategia diseñada para el mejoramiento de la gestión pública en torno al cumplimiento de los objetivos del Plan Nacional de Desarrollo, que tiene como propósito fundamental propiciar un cambio paulatino, pero radical, en las entidades del Estado hacia una nueva cultura de la gestión pública orientada a resultados, en la cual el ciudadano sea el eje del desempeño de la administración y se persiga permanentemente el mejor aprovechamiento de los recursos y la promoción del manejo de las tecnologías de gestión y desarrollo de sistemas de información. ${ }^{26}$

\section{2. 3. Decreto 2150 de 1995}

En su Artículo 26, este Decreto impone a las entidades de la Administración Pública habilitar sistemas de transmisión electrónica de datos para que los usuarios envíen o reciban información requerida en sus actuaciones frente a la Administración.

\section{2. 4. Decreto 1151 de 2008}

El Artículo 3 de esta norma establece que los principios aplicables a la estrategia de Gobierno en línea son el Gobierno centrado en el ciudadano; la visión unificada del Estado; el acceso equitativo y multicanal; la protección de la información del individuo; la credibilidad y confianza en el Gobierno en línea.

Según el Artículo 5, las fases de Gobierno en línea son:

i) Fase de información en línea: las entidades habilitan sus propios sitios web para proveer información en línea, junto con esquemas de búsqueda básica.

ii) Fase de interacción en línea: se habilita la comunicación de dos vías entre entidades y ciudadanos y empresas con las consultas en línea e interacción con servidores públicos.

26 Departamento Nacional de Planeación, Documento Conpes 2790 de 1995, Gestión pública orientada a resultados (Bogotá: Autor, 1995), 1. 
iii) Fase de transacción en línea: se proveen transacciones electrónicas para la obtención de productos y servicios.

iv) Fase de transformación en línea: se realizan cambios en la forma de operar de las entidades para organizar los servicios alrededor de necesidades de ciudadanos y empresas, con ventanillas únicas virtuales y mediante el uso de la intranet gubernamental.

v) Fase de democracia en línea: se incentiva a la ciudadanía a participar de manera activa en la toma de decisiones del Estado y la construcción de políticas públicas mediante el aprovechamiento de las tecnologías de la información y la comunicación.

\subsubsection{Ley 1341 de 2009}

Esta Ley determina el marco general para la formulación de las políticas públicas que regirán el sector de las tecnologías de la información y las comunicaciones. El octavo principio de la Ley tiene por nombre "Masificación del Gobierno en línea" y exige a las entidades públicas adoptar todas las medidas necesarias para garantizar el máximo aprovechamiento de las tecnologías de la información y las comunicaciones en el desarrollo de sus funciones.

\subsubsection{Decreto 2693 de 2012}

En su Artículo 6, esta norma enumera los temas prioritarios para avanzar en la masificación de Gobierno en línea, entre los que se encuentran: provisión de trámites y servicios por múltiples canales y uso de tecnologías de información y comunicaciones en los procedimientos administrativos (acceso a todos); interoperabilidad, cadenas de trámites y ventanillas únicas virtuales; uso eficiente de las tecnologías de la información y las comunicaciones, con el fin de mitigar el impacto ambiental; garantía de Gobierno abierto; procesos de participación, por medios electrónicos, de los ciudadanos o usuarios para construir políticas, planes, programas y proyectos, ejercer control social, resolver problemas que los afecten o tomar decisiones.

Expone en su Artículo 7 las fases del modelo Gobierno en línea, que son: la información en línea, la interacción en línea, la transacción en línea, transformación (los sujetos obligados hacen cambios en la manera de operar para eliminar límites entre sus dependencias y con otras entidades públicas e intercambian información por 
medios electrónicos con lenguaje común de intercambio de información), democracia en línea, elementos transversales (los sujetos obligados conocen sus grupos de usuarios, han identificado sus necesidades e investigan permanentemente sobre los cambios en las tendencias de comportamiento para aplicar este conocimiento en diferentes momentos de interacción), mejoramiento permanente.

\subsubsection{Decreto 2573 de 2014}

En su Artículo 4, este Decreto establece como principios de la estrategia de Gobierno en línea el debido proceso, la igualdad, imparcialidad, buena fe, moralidad, participación, responsabilidad, transparencia, publicidad, coordinación, eficacia, economía y celeridad, consagrados en los Artículos 209 de la Constitución Política, 3 de la Ley 489 de 1998 y 3 de la Ley 1437 de 2011.

Asimismo, plantea como fundamentos de la estrategia: la excelencia en el servicio al ciudadano, la apertura y reutilización de datos públicos, la estandarización (facilitar la evolución de la gestión de TI del Estado colombiano hacia un modelo estandarizado que aplica el marco de referencia de arquitectura empresarial para la gestión de TI), la interoperabilidad (fortalecer el intercambio de información entre entidades y sectores), la neutralidad tecnológica (garantizar la libre adopción de tecnologías, de acuerdo con recomendaciones, conceptos y normativas de los organismos internacionales competentes e idóneos en la materia, que permitan fomentar la eficiente prestación de servicios, emplear contenidos y aplicaciones que usen tecnologías de la información y las comunicaciones, así como garantizar la libre y leal competencia y que su adopción sea armónica con el desarrollo ambiental sostenible), la innovación (desarrollar nuevas formas de usar las tecnologías de la información y las comunicaciones) y la colaboración (implementar soluciones específicas para problemas públicos, mediante el estímulo y aprovechamiento del interés y conocimiento de la sociedad, al igual que un esfuerzo conjunto dentro de las propias entidades públicas y sus servidores).

Según el Artículo 5, los componentes de la estrategia son los siguientes:

1. TIC para servicios. Dar solución a las principales necesidades y demandas de los ciudadanos y empresas, en condiciones de calidad, facilidad de uso y mejoramiento continuo. 
2. TIC para el Gobierno abierto. Comprende las actividades encaminadas a fomentar la construcción de un Estado más transparente, participativo y colaborativo mediante el uso de las tecnologías de la información y las comunicaciones.

3. TIC para la gestión. Comprende la planeación y gestión tecnológica, la mejora de procesos internos y el intercambio de información, así como la gestión y el aprovechamiento de la información para el análisis, la toma de decisiones y el mejoramiento permanente, con un enfoque integral para una respuesta articulada de Gobierno y para hacer más eficaz la gestión administrativa entre sus instituciones.

4. Seguridad y privacidad de la información.

\subsubsection{Decreto 1078 de 2015}

Este Decreto tiene como objeto definir los lineamientos, instrumentos y plazos de la estrategia de Gobierno en línea para garantizar el máximo aprovechamiento de las tecnologías de la información y las comunicaciones.

En esta norma se evidencian los mismos principios, fundamentos y componentes de la estrategia de Gobierno en línea que en el Decreto 2573 de 2014.

\subsection{Avance en la estrategia Gobierno en línea}

Como resultado del monitoreo que debe hacerse en virtud de la Ley, se publica periódicamente el índice de Gobierno en línea, instrumento cuantitativo que muestra el estado del avance de las entidades en la implementación de la estrategia Gobierno en línea. ${ }^{27}$

27 República de Colombia, "Cómo avanza la estrategia de Gobierno en línea", http://estrategia. gobiernoenlinea. gov. co/623/w3-propertyvalue-7651. Html (acceso agosto 26, 2016). 
Gráfico 1. Uso de canales o medios electrónicos para obtener información, o realizar trámites, u obtener servicios, o presentar peticiones, quejas o reclamos o participar en la toma de decisiones

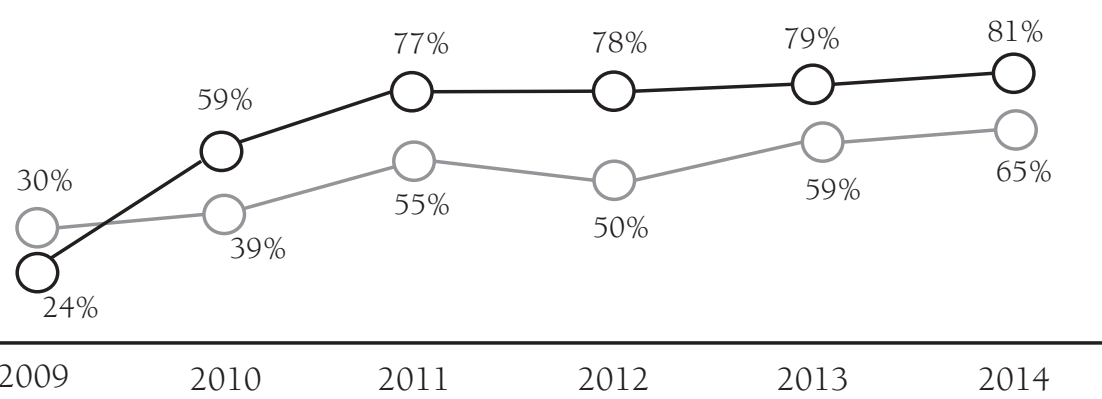

Fuente: República de Colombia, "Estrategia de Gobierno en línea. Estudios", http://estrategia.gobiernoenlinea.gov. co/623/w3-propertyvalue-7654.html (acceso agosto 26, 2016).

Según las estadísticas publicadas en la página oficial de la estrategia de Gobierno en línea representadas en el Gráfico 1, el uso de canales o medios electrónicos para obtener información, hacer trámites, obtener servicios, presentar peticiones, quejas o reclamos o participar en la toma de decisiones ha aumentado significativamente desde el año 2009, lo que demuestra que cada año el uso de las TIC está más inmerso en la relación Administración-administrado.

Gráfico 2. Estudio de cultura de uso de TIC en los colombianos para relacionarse con el Estado (2015)

\begin{tabular}{|l|c|c|c|c|}
\hline \multirow{2}{*}{$\begin{array}{l}\text { a) Obtuvieron información de las entidades públicas (horarios, } \\
\text { sedes, trámites y servicos que ofrecen, etc.) por medio de... }\end{array}$} & \multicolumn{4}{|c|}{ Durante el año 2015} \\
\hline Llamadas por teléfono fijo o celular & 27,6 & & 31,1 & \\
\hline Internet por computador de escritorio o portátil & 29,9 & 71,9 & 34,2 & 76,0 \\
\hline Internet por dispositivos móviles con celular o tablet & 14,4 & & 10,7 & \\
\hline Presencial & 28,1 & & 23,9 & \\
\hline
\end{tabular}

Fuente: MinTIC, "Estudio de cultura de uso de tic en los colombianos para relacionarse con el Estado-2015. Dirección de Gobierno en línea", http://estrategia.gobiernoenlinea.gov.co/623/articles-7913_culturadigital_2015.pdf (acceso agosto 26, 2016).

El Gráfico 2 muestra las estadísticas referentes a la relación de los ciudadanos con la Administración por medios electrónicos. Se puede observar que el porcentaje de personas que usan las llamadas telefónicas y las citas presenciales para obtener información es mayor que el de quienes utilizan Internet, lo que puede ser 
consecuencia de la baja promoción de estos medios de acceso a la información o de la alfabetización digital.

Gráfico 3. Fases de la estrategia de Gobierno en línea

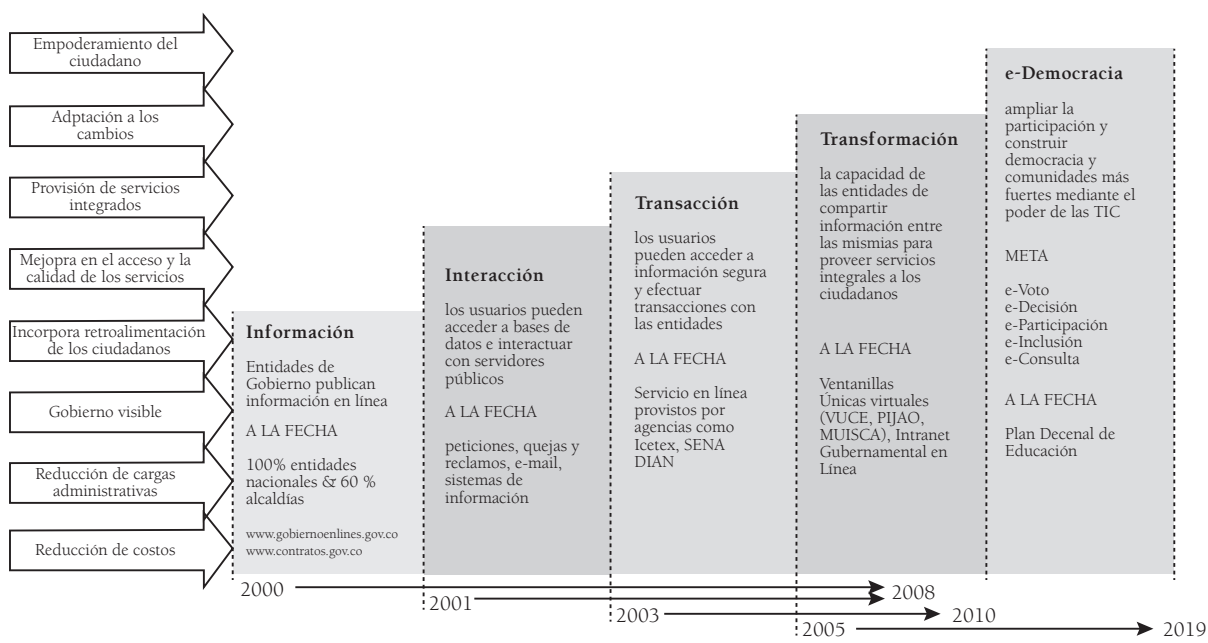

Fuente: Internet para la rendición de cuentas, "Fases de Gobierno en línea", http://iprc.org.co/concursocss/fases.html (acceso agosto 28, 2016).

Sobre las fases de la estrategia Gobierno en línea, el proyeto de Internet para la rendición de cuentas $^{28}$ representa en el esquema anterior a las fases del Gobierno en línea que se han llevado a cabo, las entidades que las han implementado y la proyección para el cumplimiento de cada etapa; asimismo, se evidencia que aunque no todas las fases han sido logradas a cabilidad, como la fase de la democracia en línea, sí se han empezado a desarrollar acciones para instituir las fases de informacion en línea y de interacción en línea.

Lo que reflejan los gráficos es que, a pesar de que se evidencia un aumento en el uso de canales o medios electrónicos para obtener información, hacer trámites, obtener servicios o presentar peticiones, quejas o reclamos o participar en la toma de decisiones, se debe mejorar la cobertura de la implementación de las TIC como medios para acceder a la información pública, con el fin de lograr el fin último de los Gobiernos electrónicos, que es "lograr la democracia y la participación electrónicas,

28 Internet para la rendición de cuentas es un proyecto que busca fortalecer la transparencia en alcaldías, entidades gubernamentales y contralorías territoriales, mediante la implementación de un software que permite el montaje de un sitio web para facilitar la entrega de información pública por Internet. 
por medio de la vinculación de la transparencia, la participación con el nexo de poderes públicos y las TIC". ${ }^{29}$

\subsection{Concepto de sistema de información y sistema informático}

Para poder definir qué son los sistemas informáticos dentro del marco de la Ley de transparencia y acceso a la información pública, primero debemos exponer qué es un sistema de información, para relacionar lo establecido en la Ley con el concepto de sistema informático. Un sistema de información es:

[...] un conjunto de procedimientos, manuales y automatizados, y de funciones dirigidas a la recogida, elaboración, evaluación, almacenamiento, recuperación, condensación y distribución de informaciones dentro de una organización, orientado a promover el flujo de las mismas desde el punto en el que se generan hasta el destinatario final de las mismas. ${ }^{30}$

Tiene entre sus funciones organizar una base de datos, de forma que se mantenga como una representación suficientemente fiable de la realidad y se facilite el acceso a partes específicas; además, presenta la información de modo que mejore la capacidad del usuario para percibir y actuar sobre los hechos reflejados por la información, proporciona una conexión esencial o interfaz entre el sistema y el usuario. Los sistemas de información tienen la función de apoyo, es decir, la que cumplen como herramientas por medio de las cuales se apoya el avance del mundo de las telecomunicaciones. ${ }^{31}$ De lo anterior se puede concluir que las funciones de los sistemas de información son el almacenamiento de datos, la presentación de la información y el apoyo a las comunicaciones.

Un sistema informático:

[...] es un sistema de información que está informatizado; es decir es un conjunto de partes o recursos formados por el hardware, software y las personas que lo emplean, que se relacionan entre sí para almacenar y procesar información con un objetivo en común. ${ }^{32}$

29 Lorenzo Cotino Hueso, Libertades, democracia y gobierno electrónicos (Granada: Comares, 2006), 179.

30 José Rodríguez y María José Daureo, Sistema de información: aspectos técnicos y legales (Almería: Universidad de Almería, 2003), 31.

31 Rodríguez y Daureo, Sistema de información, 32.

32 Alegsa, "Diccionario de informática y Tecnología. Definición de sistema informático", http://www.alegsa. com.ar/Diccionario/diccionario.php (acceso septiembre 2, 2016). 
Entonces, un sistema informático es un sistema que utiliza las tecnologías como medio de presentación de la información. En relación con el objeto de la Ley de transparencia y acceso a la información, en especial con el literal d del Artículo 17, se acopla a la exigencia de alineación con la estrategia de Gobierno en línea. Por eso, se propone que en vez de llamarles sistemas de información a los medios condicionados que presenta la Ley 1712 a los sujetos obligados, se les denomine sistemas informáticos.

Los sistemas de información, en el marco de la Ley de transparencia y acceso, son sistemas informáticos, es decir, un conjunto de procedimientos informatizados que utilizan los sujetos obligados, con el fin de recoger, elaborar, evaluar, almacenar, recuperar, condensar, difundir la información y promover el flujo de la misma.

\subsection{Implementación de sistemas informáticos para la protección del derecho al acceso a la información pública}

Vivimos en un mundo en donde el uso de las tecnologías está inmerso en las relaciones sociales, por lo que era de esperarse que los sistemas informáticos cobraran un papel importante en la relación Administración-administrados, para mejorar la comunicación entre estos y permitir que los ciudadanos accedan a la información, conozcan las actuaciones de la Administración y logren la tan anhelada transparencia que ha buscado nuestro país a lo largo de la historia.

Respecto al uso de los sistemas informáticos para la protección del acceso a la información pública, algunos doctrinantes de España, Brasil y Chile reconocen la importancia del uso de los sistemas informáticos en pro de la garantía del acceso a la información. Citaremos dos pronunciamientos. En el libro La Ley de administración electrónica, publicado en 2008 en España, se dice:

El Principio de neutralidad tecnológica y la adaptabilidad al progreso de las técnicas y sistemas de comunicaciones electrónicas es indispensable para la aplicación efectiva de la ley de administración electrónica pues depende de este que a todos los administrados se les garantice el derecho a la interacción electrónica y al acceso a la administración electrónica. ${ }^{33}$

33 Gamero Casado y Valero Torrijos, coords. , La Ley de administración electrónica, 97 y 104. 
De ahí se deduce que la adaptación de sistemas modernos y progresivos que posibilitan la comunicación entre la Administración y los administrados facilita garantizar el acceso a la información pública, por lo que la implementación de sistemas de información sistematizados amplía la cobertura de la población, para que personas a las que se les dificultaba informarse por medios presenciales puedan acceder a la información por sistemas tecnológicos.

El libro Direito e questões tecnológicas aplicados no desenvolvimento social, publicado en 2012 en Brasil, expresa las exigencias interdisciplinarias del uso de sistemas informáticos por la Administración. Manifiesta: "El uso de las tecnologías en la interacción con la administración pública requiere no solo de conocimientos jurídicos, este campo necesita la iteración interdisciplinaria con áreas como la ingeniería, la de las comunicaciones y sistemas". ${ }^{34}$ Cabe resaltar que en Colombia, para que un sistema informático garantice el derecho de acceso a la información, no solo requiere el esfuerzo de un grupo interdisciplinario, sino que debe cumplir con las exigencias del Artículo 17 de la Ley 1712 de 2014, entre las que se encuentra la alineación con la estrategia de Gobierno en línea.

\section{Alineación del sistema informático con la estrategia del Gobierno en línea}

La Ley 1712 de 2014 faculta a la Administración para implementar sistemas informáticos con el fin de permitir el acceso a la información y facilitar la interacción con la ciudadanía, pero esta facultad conlleva las obligaciones que se expusieron en el capítulo 2 del presente artículo, cuyo objetivo es garantizar que el sistema utilizado sea considerado efectivamente una herramienta para promover el acceso a la información pública.

Entre estas exigencias se encuentra la de que el sistema debe estar alineado con la estrategia de Gobierno en línea o con el que haga sus veces, obligación que es vital, pues su cumplimiento, en conjunto con las otras exigencias, permite la garantía del derecho al acceso a la información.

Puesto que en el marco de la Ley 1712 de 2014 los sistemas de información son un conjunto de procedimientos informatizados que utilizan los sujetos obligados con

34 Antônio Carlos Efing y Cinthia Obladen de Almendra Freitas, Direito e questões tecnológicas aplicados no desenvolvimento social. Vol. 2 (Curitiba: Juruá, 2012), 402. 
el fin de recoger, elaborar, evaluar, almacenar, recuperar, condensar y distribuir la información y promover el flujo de la misma, debemos preguntarnos ipor qué la Ley exige que estos se encuentren alineados con la estrategia Gobierno en línea?

Encontramos respuesta al comparar las nociones de sistema informático y de gobierno electrónico. Al analizar el concepto de sistema informático, vemos que es una forma de organizar, almacenar y poner a disposición de los interesados la información de manera electrónica, sin un fin u objetivo específico del uso de la herramienta; por el contrario, el concepto de los sistemas informáticos en el gobierno electrónico sí muestra un objetivo: "mejorar los servicios públicos, los procesos democráticos y las políticas públicas", ${ }^{35}$ con miras a lograr un Gobierno más eficiente y transparente que promueva la participación ciudadana.

Por lo tanto, la exigencia de alineación se ve fundamentada en que los sistemas informáticos implementados por los sujetos obligados no solo deben ser utilizados para administrar la información, sino que deben ser elaborados con el objetivo de mejorar los servicios públicos, los procesos democráticos y las políticas públicas mediante las tecnologías de la información y comunicación, como lo plantea nuestra estrategia de gobierno electrónico denominada Gobierno en línea.

Tras reconocer el fundamento de la exigencia impuesta por ley y hacer un recuento normativo de la estrategia de Gobierno en línea, plantearemos los siguientes lineamientos que debe tener en cuenta el sujeto obligado en el momento de implementar su sistema informático para que este se encuentre alineado con la estrategia de Gobierno en línea:

- $\quad$ El sistema debe tener como propósito la presentación de servicios de calidad en línea para ofrecer excelencia en la gestión pública por medio del uso estratégico de las TIC.

- Debe tener en cuenta el respeto y la seguridad con los que se deben tratar los datos de los ciudadanos y su privacidad.

- El sistema también debe estar fundamentado en los principios del debido proceso, igualdad, imparcialidad, buena fe, moralidad, participación, responsabilidad,

35 Comisión de las Comunidades Europeas, Comunicación de la Comisión sobre el papel de la administración electrónica en el futuro de Europa (Bruselas, 26 de septiembre de 2003). 
transparencia, publicidad, coordinación, eficacia, economía y celeridad consagrados en los Artículos 209 de la Constitución Política, 3 de la Ley 489 de 1998 y 3 de la Ley 1437 de 2011.

El sistema debe respetar los principios aplicables a la estrategia de Gobierno en línea, por lo que deberá: i) estar centrado en el ciudadano, permitirle un acceso equitativo y multicanal, proteger la información del individuo y generar credibilidad y confianza en el sistema y en la Administración misma; ii) reutilizar datos públicos y, en virtud del principio de estandarización, facilitar la evolución de la gestión de tecnologías de la información del Estado colombiano hacia un modelo estandarizado; iii) fortalecer el intercambio de información entre entidades y sectores, dado el principio de interoperabilidad, garantizar la libre adopción de tecnologías en pro de la neutralidad tecnológica e innovar con miras al desarrollo de nuevas formas de usar las tecnologías de la información y las comunicaciones, y iv) cumplir el principio de colaboración, que implica la implementación de soluciones específicas para problemas públicos, mediante el estímulo del interés y conocimiento de la sociedad, con la ayuda del esfuerzo conjunto de las propias entidades públicas y sus servidores.

- De acuerdo con las fases de la estrategia de Gobierno en línea inscritas en el Decreto 1151 de 2008, la herramienta que utilice el sujeto debe publicar la información en línea, permitir que los ciudadanos interactúen con la Admistración, facilitar la realización de transacciones en línea con la entidad —si a eso hubiera lugar - y tener como meta la participación e inclusión del administrado por vía electrónica.

- El sistema debe tener en cuenta los tres componentes de la estrategia de Gobierno en línea: TIC para servicios, TIC para Gobierno abierto, TIC para la gestión y seguridad y privacidad de la información. El sistema tiene que dar solución a las principales necesidades y demandas de ciudadanos y empresas en condiciones de calidad, facilidad de uso y mejoramiento continuo; estar encaminado a fomentar la construcción de un Estado más transparente, participativo y colaborativo mediante el uso de las tecnologías de la información y las comunicaciones; comprender la planeación y gestión tecnológica, la mejora de procesos internos y el intercambio de información; por último, garantizar la seguridad y privacidad de la información. 
Como se puede observar, las exigencias que debe cumplir el sujeto obligado para que su sistema se encuentre alineado con el Gobierno en línea están relacionadas con el objetivo y los principios de la estrategia, que se resumen en la utilización de las TIC como medio para permitir el acceso a la información y la participación ciudadana y como herramientas para mejorar la gestión pública.

Sin embargo, al revisar los lineamentos que tendría que tener en cuenta el sujeto obligado en el momento de implementar su sistema, se concluye que para cumplir con el objetivo de la norma y evitar que el sistema no está acorde con la estrategia de Gobierno en línea, sería más práctico crear un sistema informático que cumpla con todas las exigencias de la Ley y difundirlo entre los sujetos obligados. De esta forma, no solo se garantizaría el derecho de acceso a la información, sino que todos los sujetos obligados por medio de un "sistema único de acceso" harían efectivo este derecho.

Por otra parte, permitir que los sujetos obligados utilicen sus propios sistemas de información y dejar a su discreción el cumplimiento de los requerimientos que la Ley impone genera el riesgo de que lo contemplado por la Ley se quede en un ideal formalizado legalmente y no se materialice. De lo anterior surge un nuevo problema jurídico respecto a iqué podría hacer un ciudadano ante el hecho de que un sujeto obligado no alinee su sistema con la estrategia de Gobierno en línea?

En principio, podría plantearse, la posibilidad de tutelar esta situación como consecuencia de que el sistema empleado por los sujetos obligados es el medio para la garantía del derecho de acceso a la información que, en virtud de la Ley 1712 de 2014, tiene carácter de derecho fundamental autónomo; no obstante, la importancia de este problema jurídico requiere una investigación más profunda para dar una respuesta sustentada a esta nueva pregunta que surge de la reflexión expuesta en este artículo.

\section{Conclusiones}

- "La información pública deriva de múltiples disposiciones, entre las que se encuentra la publicidad de los actos y las normas". ${ }^{36}$ La importancia del derecho a acceder a ella radica en su papel como instrumento para la consagración de otros derechos, su uso para prevenir la corrupción, su importancia como medio

36 Ruiz-Rico, Tendencias legales, 13. 
de control de la actuación de la Administración y su función como medio para garantizar la participación, la democracia y la transparencia.

- Respecto al concepto de sistemas de información consagrado en la Ley 1712 de 2014, se propone que en su lugar utilice el concepto de "sistemas informáticos", toda vez que del resultado del análisis del concepto de sistemas de información en el marco de la misma Ley se concluyó que este sería el término apropiado. Esto como consecuencia tanto del incentivo del uso de las tecnologías por la Ley como de la misma exigencia de alineación con el Gobierno en línea, que hace que los sistemas usen y promuevan el desarrollo de las tecnologías de la información.

- Las exigencias que deben cumplir los sujetos obligados para que su sistema informático cumpla con la obligación de alineación con la estrategia de Gobierno en línea están estrechamente relacionadas con el objetivo del gobierno electrónico de "mejorar los servicios públicos, los procesos democráticos y las políticas públicas", ${ }^{37}$ con miras a lograr un Gobierno más eficiente y transparente y con la participación ciudadana. Con el propósito de cumplir este objetivo, los sistemas informáticos deben estar acordes con los principios de la Administración pública y los propios del Gobierno en línea, lo que se resume en el cumplimiento de los componentes de la estrategia.

Para cumplir con los componentes del Gobierno en línea, el sistema debe dar solución a las principales necesidades y demandas de los ciudadanos y empresas en condiciones de calidad, facilidad de uso y mejoramiento continuo; estar encaminado a fomentar la construcción de un Estado más transparente, participativo y colaborativo mediante el uso de las tecnologías de la información y las comunicaciones; comprender la planeación y gestión tecnológica, la mejora de procesos internos y el intercambio de información; por último, garantizar la seguridad y privacidad de la información.

Al ser tantos los lineamientos que el sujeto debe tener en cuenta, se propone la creación y difusión de un sistema de información que cumpla con todas las exigencias de la ley 1712 de 2014, un "sistema único de acceso" implementado por todos los sujetos obligados, para hacer efectivo el derecho de acceso a la información pública.

37 Comisión de las Comunidades Europeas, Comunicación de la Comisión sobre el papel de la administración electrónica. 


\section{Referencias}

Alegsa. "Diccionario de informática y Tecnología. Definición de sistema informático". http:// www. alegsa. com. ar/Diccionario/diccionario. php (acceso septiembre 2, 2016).

Andrade Castro, Jesús y Yedra Hernández Yaskelly. Sistemas Transparentes para Gobiernos Electrónicos Eficientes. Maracaibo: Red Enlace, 2007.

Becerra Jairo, Germán Darío Flórez Acero, Claudia García Vargas, Carolina Rojas Orjuela Vargas, Marco Emilio Sánchez Acevedo, Jheison Torres Ávila. El Derecho y las tecnologías de la información y la comunicación (TIC). Bogotá: Universidad Católica de Colombia, 2015.

Cobo Romaní, Juan Cristóbal. "El concepto de tecnologías de la información. Benchmarking sobre las definiciones de las TIC en la sociedad del conocimiento". Zer: Revista de estudios de comunicación 14, núm. 27(2009): 295-318.

Comisión de las Comunidades Europeas. Comunicación de la Comisión sobre el papel de la administración electrónica en el futuro de Europa. Bruselas, 26 de septiembre de 2003.

Congreso de la República Colombia. Ley 57 de 1985, "Por la cual se ordena la publicidad de los actos y documentos oficiales". Bogotá: Diario Oficial, 5 de julio de 1985.

Cotino Hueso, Lorenzo. Libertades, democracia y gobierno electrónicos. Granada: Comares, 2006.

Cotino Hueso, Lorenzo. "Derechos fundamentales de acceso a la información pública y participación ciudadana, regulación constitucional, estatutaria y legal". Ponencia presentada en el seminario El Gobierno abierto en el Estatuto de Autonomía y su desarrollo. Especial atención a la futura ley de transparencia, buen gobierno y participación ciudadana de la Comunitat Valenciana, Valencia, 30 de septiembre, 2014.

Cotino Hueso, Lorenzo. "Teoría y realidad de la transparencia pública en Europa". http:// docplayer.es/1 1550709-Teoria-y-realidad-de-la-transparencia-publica-en-europalorenzo-cotino-hueso-2003.html (acceso agosto 25, 2016).

Departamento Nacional de Planeación. Documento Conpes 2790 de 1995, Gestión pública orientada a resultados. Bogotá: Autor, 1995.

Efing,Antônio Carlos y Cinthia Almendra Freitas. Direito e questões tecnológicas aplicados no desenvolvimento social. Vol. 2. Curitiba: Juruá, 2012.

Federación Iberoamericana de Ombudsman. XIII Informe sobre derechos humanos. transparencia e información pública. Madrid: Trama editorial,2015.

Gamero Casado, Eduardo y Juliana Valero Torrijos, coords. La Ley de administración electrónica. Pamplona: Aranzadi, 2008.

Internet para la rendición de cuentas, "Fases de Gobierno en línea". http://iprc.org.co/ concursocss/fases.html (acceso agosto 28, 2016). 
MinTIC. "Boletín trimestral de las TIC. Cifras primer trimestre 2016)". http://colombiatic. mintic.gov.co/602/articles-15639_archivo_pdf (acceso agosto 3, 2016).

MinTIC. "Estudio de cultura de uso de tic en los colombianos para relacionarse con el Estado-2015. Dirección de Gobierno en línea". http://estrategia.gobiernoenlinea.gov. co/623/articles-7913_culturadigital_2015.pdf (acceso agosto 26, 2016).

Nava, Salvador, Issa Luna y ErnestoVillanueva. Derecho de acceso a la información pública parlamentaria. Ciudad de México: Porrúa, 2006.

Organización de Estados Americanos [OEA]. "Relatoría especial para la libertad de expresión 2007". http://cidh.oas.org/relatoria/section/Estudio\%20Especial\%20 sobre\%20el\%20derecho\%20de\%20Acceso\%20a\%20la\%20Informacion.pdf (acceso julio 20, 2016).

Orszag, Peter R. "Memorandum for the Heads of Executive Departments and Agencies, de 8 de diciembre de 2009". https:/www. whitehouse.gov/open/documents/open-government-directive (acceso agosto 17, 2016).

Porro Gutiérrez, Jacinto. Instrumentos y procesos de participación ciudadana en España y Marruecos. Madrid: Dykinson, 2012.

Ramírez, José, Deslisireth Rincón y María Romero. "Gobierno electrónico: un signo de inclusión digital y poder popular". Revista de Ciencias Sociales 16, núm 4 (2010): 709-720. República de Colombia. Constitución Política. Bogotá: Legis, 1991.

República de Colombia, Estrategia de Gobierno en Línea "Estudios" http://estrategia. gobiernoenlinea.gov.co/623/w3-propertyvalue-7654.html (acceso agosto 26, 2016). República de Colombia. "Cómo avanza la estrategia de Gobierno en línea". http://estrategia. gobiernoenlinea.gov.co/623/w3-propertyvalue-7651.html (acceso agosto 26, 2016). República de Colombia. "Declaración de compromiso con la agenda post 2015" (2015). —ODS". http://estrategia.gobiernoenlinea.gov.co/623/articles-7929_agenda_ods.pdf (acceso julio 26, 2016).

República de Colombia. "Estrategia de Gobierno en línea”. http://estrategia.gobiernoenlinea. gov.co/623/w3-propertyvalue-7650.html (acceso agosto 26, 2016).

Rodríguez, José y María José Daureo. Sistema de información: aspectos técnicos y legales. Almería: Universidad de Almería, 2003.

Ruiz-Rico, Catalina. Tendencias legales hacia la calidad democrática del sistema constitucional. Madrid: Dykinson,2014.

The Carter Center. Access to Information:A Key to Democracy. Atlanta: Autor, 2002.

The White House. "Open Government Initiative". https://www.whitehouse.gov/open (acceso agosto 17, 2016).

Torres Díaz, María Concepción. "La fundamentalidad del derecho de acceso a la información pública: análisis crítico desde el marco constitucional de la participación ciudadana" en El paradigma del Gobierno abierto. Retos y oportunidades de la participación, transparencia 
y colaboración, editado por Lorenzo Cotino, José Luis Sahuquillo y Loreto Corredoira. Madrid: Universidad Complutense de Madrid y ObservatorioTIC-Cyberlaw Clinic, 2015. Villanueva, Ernesto. Derecho de acceso a la información pública en Latinoamérica. Ciudad de México: Instituto de Investigaciones Jurídicas, UNAM, 2004. 